\title{
CONSTRUCTION OF INTELLIGENT CONTROL DECISION SUPPORT SYSTEM IN ENTERPRISES
}

\author{
Zheng Liu ${ }^{1}$, Qiong Sun ${ }^{*}$ \\ $1,2^{*}$ Beijing Union University, \\ Beijing 100101, China \\ E-mail: zhenglzlz@sina.com, sunqiongsqsq@163.com
}

\begin{abstract}
The constant advancement of network makes the life and work of people more highefficient and easier. Enterprises should make decision by scientifically and accurately analyzing mass data on the internet. Therefore it is urgent to establish a system which can support enterprise decision-making. Intelligent decision support system can integrate relevant theories such as management, operation, overall planning and control with behavioural science and intelligently support decision-making through computer equipment, technology and simulation. Based on complex network system, this study set data acquisition, data analysis, data mining, enterprise trade, performance evaluation and profit as six subsystems and set up a commercial intelligent decision support system. Finally simulation verification was performed on the system. The results demonstrated that the system had a reliability of 0.9889 , suggesting the decision support system had a high accuracy, and the system could effectively support the decision making of enterprises. In conclusion, the decision support system has certain feasibility, which is worth promotion and application.
\end{abstract}

Keywords: Intelligent Control, Decision Support System, Complex Network, Intelligent Decision Support System.

\section{Introduction}

In nowadays society, studies concerning science and technology such as internet, cloud computation and database are developing rapidly, which has great impacts on the establishment of enterprises. Various data will be generated in the production and operation of enterprises, and a system which can extract effective information from various data can help enterprises make scientific decision [1].

Decision support system is an information system and also an interactive computer system. It supports the decision of decision makers through man-machine conversation and graphics part based on database, knowledge database, model base and method base [2, 3].

Intelligent control decision support system mainly includes man-machine interactive system, database system, knowledge base system and model base system [4].

Intelligent decision support system has been extensively applied. Fernandes et al. [5] used intelligent decision support system in network news to extract the keywords of an article and digital media content, then predicted the popularity of articles using intelligent decision support system and optimized article character subset which was easy to be modified.
The average optimization gain increased for $15 \%$, suggesting the intelligent decision support system had a preferable support for the scientific decision of network media. Sellak et al. [6] proposed an intelligent re-dss framework to plan and design intelligent energy management system.

The framework provided an extensively applied classical re-dss, which helped project management at different levels make high-efficient decision. Dovbysh et al. [7] put forward to observe the information synthesis pattern of a human organ radionuclide diagnosis decision support system with $\gamma$ ray dynamics and optimized the problem of dividing characteristic space into class equivalent geometrical parameter in the process of information ability maximization of decision support system.

This study found that the reliability of the decision support system was 0.9889 , suggesting a high accuracy and a strong support for enterprise decision.

\section{Optimal Configuration under Complex Network Environment}

In enterprise operation and management, different subsystems will be abstracted to be network systems. Such network systems are the components of complex network system. 
System configuration optimization under complex network environment can further improve the overall reliability of the whole system and the utilization of energy $[8,9]$.

Generally, the expense of enterprise operation and management will not increase infinitely, but within a certain control range. Hence it is the most important to control expense under an acceptable scope and control the decisions of operation and management to the largest extent.

The resource quantity of different subsystems was regarded as the variables of the whole model. To control the total expense within the reasonable scope, suppose

$$
\begin{gathered}
t=\max A=\max T\left(A_{1}, A_{2}, \ldots \ldots, A_{\mathrm{n}}\right) \\
\left\{\begin{array}{c}
\sum_{i=1}^{n} W_{i} \times X_{i} \leq W,(i=1,2, \ldots \ldots, n) \\
\mathrm{H}_{\mathrm{i}} \leq A_{i} \leq Q_{i},(i=1,2, \ldots \ldots, n) \\
1 \leq X_{i} \leq N,(i=1,2, \ldots \ldots, n)
\end{array}\right.
\end{gathered}
$$

(2) included three constraint conditions. When the constraint conditions of (2) were satisfied, $A_{1}, A_{2}, \ldots \ldots, A_{\mathrm{n}}$ were calculated, which could maximize the reliability of decision $t . W_{i}$ stands for the expense of every subsystem, $W$ stands for the upper limit of total expense, $A$ stands for the decision reliability of different subsystems, $t$ stands for total decision reliability, $Q_{i}$ stands for the highest reliability of decision, $\mathrm{H}_{\mathrm{i}}$ stands for the lowest reliability, and $N$ stands for the upper limit of allocable resource. The flow chart [10] is shown in Figure 1.

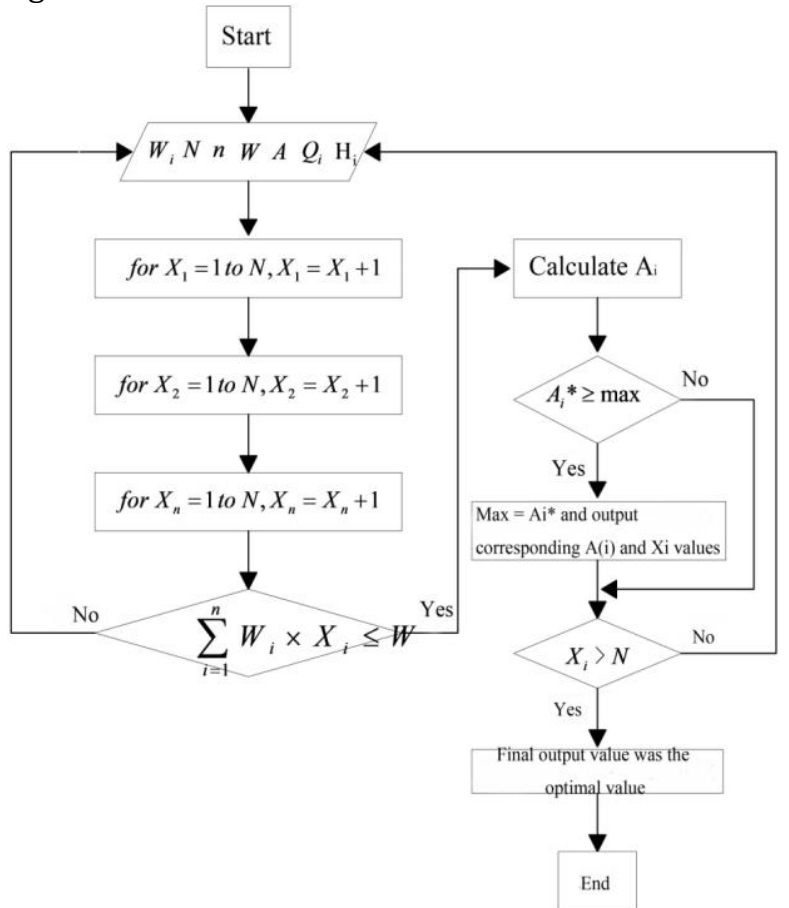

Figure 1: The optimal flow under complex network
To make the system more optimal and reliable in enterprise decision-making in complex network environment, the feasible solutions which satisfied all conditions $\left(X_{1}, X_{2}, X_{3}, \ldots, X_{\mathrm{n}}\right)$ and reliability $\left(A_{1}, A_{2}, A_{3}, \ldots, A_{\mathrm{n}}\right)$ were calculated through computer program, then the reliability of the system $T\left(A_{1}, A_{2}, A_{3}, \ldots, A_{\mathrm{n}}\right)$, and the result obtained after comparison was the optimal solution of $\operatorname{Max} T\left(A_{1}, A_{2}, A_{3}, \ldots, A_{\mathrm{n}}\right)$.

\section{Construction of Business Intelligent Decision Support System}

Because of enterprise information overload and the constant development of information technology, business intelligence visits and shares data with the opposite side on supply chain. Business intelligence can not only collect the information generated by enterprises, but also develop and process information and transform them to data which can support enterprise decision-making $[11,12]$. Hence this study focused on constructing enterprise account intelligent decision support system.

As shown in Figure 2, the operation of a business intelligent decision support system cannot be separated from decision-maker, man-machine interaction and database subsystem. Considering the optimal configuration of complex network, this study established data acquisition, processing and analysis subsystems, trade subsystem, performance evaluation subsystem and decision support subsystem.

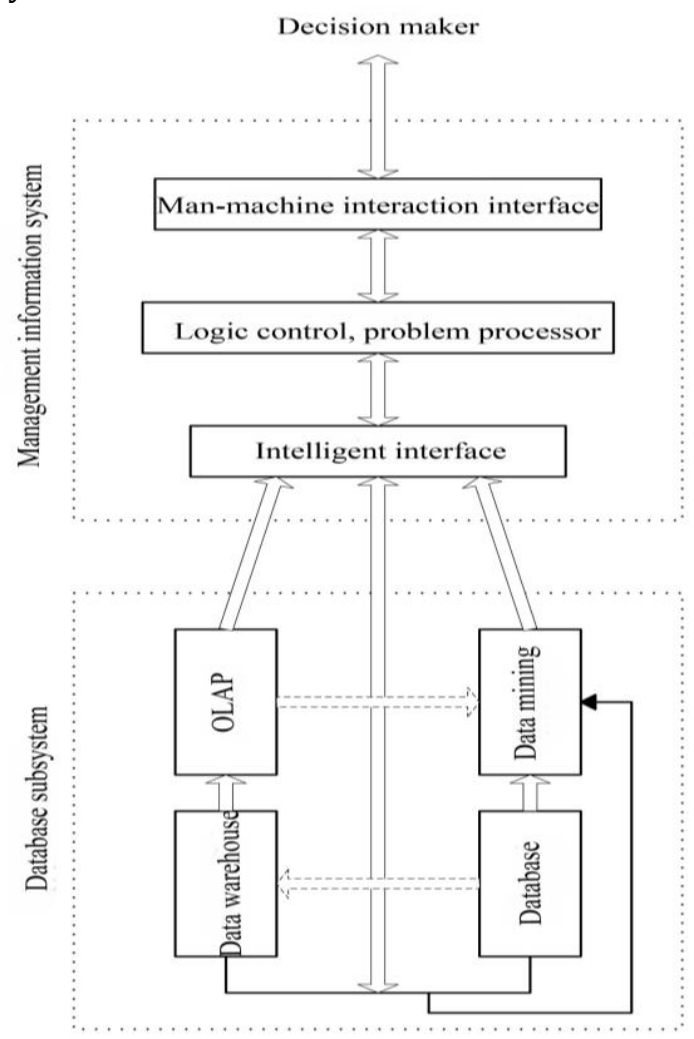

Figure 2: The profile of business intelligence system 


\subsection{Establishment of data acquisition subsystem}

Generally, data collection degree directly affects the integrity of a database. Hence the comprehensiveness and accuracy should be noticed in data acquisition. As some data have features such as heterogeneous platform, for example, data of rivals and market data of suppliers, requirements on collection tools and network are high. Information inside and outside enterprises were gathered by Web site, Internet resource and enterprise Internet.

\subsection{Design of data analysis subsystems}

Data processing mainly includes conversion and integration. Data were converted according to unified conversion code. For example, male client could be converted to 1 and female client could be converted to 0 . As to data integration, data were coordinated and sorted according to structured, semi-structured and non-structured patterns to ensure the consistency of customer number and recognition system. Therefore, the customer information from different departments of an enterprise should be imported to the data warehouse of the enterprise.

\subsection{Establishment of data mining subsystem}

The first step of establishing database subsystem was to collect and convert data, input data to data warehouse using ETL tool and establish multidimensional model and data mining with data warehouse. Data mining was to mine potential data from a large amount of data. OLAP technology was used in this study.

OLAP technology classified the attributes of data into dimension and measurement. Finally, data were displayed. Due to the limitations of time and capital, Microsoft SQL Server 2005 platform was used to establish database subsystem. Data warehouse was established using SQL Server Management Studio (SQL SMS), and OLAP, data mining and reports used SQL Server Business Intelligence Development Studio (SQL SBIDS).

\subsection{Design of trade subsystem}

It was simulated trade; hence an enterprise can directly process business matters with other enterprises instead of using actual capital during simulated trade. Firstly the requirements are analyzed. Requirement analysis should consider risk factors such as laws, politics, market and finance. All target enterprises which were suitable for trade were listed. Then the values of the target enterprises were assessed; finally the trading enterprises were selected. Next the values and total values of the enterprise were further evaluated. After requirement analysis, the collected data were analyzed.

The implementation procedures of the trade system were as follows.

Firstly, the enterprise was analyzed. The requirements and motives of the enterprises were determined and relevant data were input to the system to determine the values of the enterprises.

Secondly, the trading object was determined. Similarly, relevant data were input to the system to determine its value.

Thirdly, the system evaluated post-trade values automatically in background according to cost analysis and profit decision making.

Based on the final value evaluation of all the enterprises, the optimal trading object was selected for simulated trade.

\subsection{Performance evaluation subsystem}

Performance evaluation subsystem plays roles in the achievement of anticipated performance and the evaluation of actual performance. This study firstly determined the possible factors influencing performance and selected corresponding models for evaluation. Then the performance evaluation subsystem was connected with the data acquisition, processing and analysis subsystems.

To judge anticipated and actual performance, the model base and knowledge base were connected.

The system input the needed data to the model to obtain the final result. Both quantitative and qualitative results could support the final decision.

\subsection{Profit decision-making subsystem}

The decision-making procedures of profit decision-making subsystem were as follows. Firstly, the name of decision was input; then the decisionmaking model was selected from model base; the decision-making conditions were selected and added to generate SQL condition sentence. Decision-making content was selected for target conditions.

As it was profit decision-making subsystem, profit value was regarded as the target number. User clicked the button of "operation", and the system output analysis results. If users wanted to input decision-making objectives again, they could change conditions to make new decisions or change analysis conditions to process and analyze decision-making objectives.

\section{Analog Computation Experiment}

Decision-making analysis was made on profit, cost, sales and market. Then the subsystem was put in a complex network to search for optimal configuration and analyze the support for enterprise decision- 
making. The data acquisition subsystem, data analysis subsystem, data mining subsystem, trade subsystem, performance evaluation subsystem and profit subsystem were set as system $1 \sim 6$.

Moreover, the lower and upper limit of reliability of every subsystem was 0.5 and 0.95 respectively. The upper limit of total cost was 500 yuan.

\section{Results}

\subsection{Profit decision analysis}

Special steel plate produced by an enterprise was taken as an example. The sales price table of an enterprise suggested that, when the price was 9500 yuan/t, the sales quantity was 100 ton; when it was 9000 yuan/ton, the sales quantity was 500 ton; when it was 8500 yuan/t, the sales quantity was 550 t. the cost was 7500 yuan/t.

When the profit was the largest, the price was 8880 .

\subsection{Cost decision analysis}

The cost decision of an enterprise is to determine the sum of standard cost, do cost plus and obtain market price. Generally, cost includes manufacturing cost, variable manufacturing overhead expenses, material price and salary adjustment expense.

Cost price and reduction rate are compared with target reduction amount and reduction rate. If the results are close, it indicates that target cost is guaranteed to some extent. If the decision result is far away from the target, it indicates that the potential of reducing cost remains to be explored, and decision-making should be performed again.

For example, the target cost of an enterprise was 6000 yuan/t, and the decision result was 6300 yuan/t; then the two data were close.

\subsection{Market decision analysis}

Market decision analysis means analyzing the similar products in market in aspects of price and influence and formulating suitable sales market strategies for the products of an enterprise. Market decision analysis of an enterprise is to analyze consumers in the aspects of market characteristics, consumption population and economic income, predict the life cycle and sales time of products, make decision-making analysis on the distribution of product demand areas and category of products and formulate relevant strategies.

\subsection{Optimal configuration}

Table 1 demonstrates that, in the complex network environment and after optimal configuration, when the total system cost was 465 yuan, the reliability of the system was 0.9889 .

The reliability of the system could reflect the accuracy of the decision; the system provided support for the decision.

Table 1: The optimal configuration of the decision

\begin{tabular}{|c|c|c|c|}
\hline \multicolumn{4}{|c|}{ support system } \\
\hline No. of subsystem & $X_{i}$ & $A_{i}$ & $W_{i} X_{i} /$ yuan \\
\hline 1 & 3 & 0.9281 & 74 \\
\hline 2 & 4 & 0.9374 & 121 \\
\hline 3 & 1 & 0.8020 & 19 \\
\hline 4 & 2 & 0.9101 & 41 \\
\hline 5 & 1 & 0.8020 & 19 \\
\hline 6 & 3 & 0.9281 & 74 \\
\hline $\begin{array}{c}\text { Total system cost } \\
\text { W/ yuan }\end{array}$ & & 46 & \\
\hline $\begin{array}{c}\text { Objective } \\
\text { function value }\end{array}$ & 0.98 & & \\
\hline $\begin{array}{l}\text { Constraint } \\
\text { condition }\end{array}$ & & $\begin{array}{c}<500 \\
H_{i}<\end{array}$ & $\begin{array}{l}X_{i}<5, \\
<Q_{i}\end{array}$ \\
\hline
\end{tabular}

\section{Discussion and Conclusion}

In the trend of constant development, it is necessary to improve enterprise competitiveness using business intelligent decision support system.

Due to the limitation of technology, the fast updating of network data and complex environment, there are defects in data analysis and mining.

To improve the operation ability and decisionmaking level of enterprises, decision support system should be established to help enterprises extract useful information through integrating and analyzing relevant data and increase the scientificity of decision. Brandao et al. [13] established obstetrical nursing decision support system, designed two data warehouses with different dimension, and proposed the architecture of BI solution, which was of important significance to the health of patients and reduction of medical errors.

Sun et al. [14] considered that BI system was mainly composed of data warehouse, ETL, OLAP, data mining and front end data visualized technology and established a mashup framework based double front end tool architecture based on the advantages of mashup technology in the development of selfservice application program. 
In the implementation process of decision support system, useful information was extracted from static data source; the information was transformed to abstract knowledge which can be used for decision and help decision-maker make correct decision. Because of the openness of network, the mode of information utilization changes greatly, and information is no longer open to some individuals $[15,16]$. In such a background, seizing the opportunity is extremely beneficial to the development of enterprises.

This study designed data acquisition subsystem, data analysis subsystem, data mining subsystem, trade subsystem, performance evaluation subsystem and profit subsystem and found that the reliability of the decision support system was 0.9889 , indicating the high accuracy of the decision support system.

The system can effectively support the decision of enterprises; hence it is worth extensive application.

\section{Acknowledgements}

This study was supported by the General Project of the Beijng Municipal Education Committee's Scientific and Technological Plan (KM201711417013) to Sun Qiong.

\section{References}

[1] Kar A K. "A hybrid group decision support system for supplier selection using analytic hierarchy process. fuzzy set theory and neural network," J. Comput. Sci., 2015, 6:23-33.

[2] Wenkel K O, Berg M, Mirschel W, Wieland R, Nendel C, Köstner B. "LandCaRe DSS--an interactive decision support system for climate change impact assessment and the analysis of potential agricultural land use adaptation strategies," J. Environm. Manag., 2013, 127(3):S168.

[3] Por L Y, Boey R F, Ang T F, Liew C S. "An interactive web-based wedding planner with comparative analysis decision support system. WSEAS Transact," Informat. Sci. Appl., 2008, 5(3):211-220.

[4] Anzaldi G, Rubion E, Corchero A, Sanfeliu R, Domingo $\mathrm{X}$, Pijuan J, Tersa F. "Towards an Enhanced Knowledge-based Decision Support System (DSS) for Integrated Water Resource Management (IWRM)," Proc. Eng., 2014, 89:1097-1104.
[5] Fernandes K, Vinagre P, Cortez P. "A Proactive Intelligent Decision Support System for Predicting the Popularity of Online News," 2015, 9273:535-546.

[6] Sellak H, Ouhbi B, Frikh B. "Towards an Intelligent Decision Support System for Renewable Energy management," International Conference on Intelligent Systems Design and Applications, IEEE, 2015:319-324.

[7] Dovbysh A S, Moskalenko V V, Rizhova A S, Dyomin $\mathrm{O}$ V. "Intelligent Decision Support System for Medical Radioisotope Diagnostics with Gamma-camera," J. Market. Res., 2015 7(4):608-627.

[8] Brailas, N M, Kindle B J, Sharif-Homayoun A, Rivard W G. "Systems and methods for operating and management of RFID network devices: US, US 20060280181 A1," 2006.

[9] Fujisawa S, Takeshita H, Hino T, Tajima A. "Distance-adaptive spectrum slot allocation algorithm for efficient use of network resources on elastic optical network," 19th Optoelectronics and Communications Conference (OECC) and the 39th Australian Conference on Optical Fibre Technology (ACOFT). Eng. Austral, 2014:541-542.

[10] Kong G, Yu Y. "Necessities to Strengthen the Analysis of Human Reliability," J. Univ. Electr. Sci. Technol. Chin., 2002, 31(2):200-203.

[11] Varella L, Gonçalves M B. "Information Technology as the Main Competence in the Design of the Strategic Planning of Logistics Platforms," J. Tech. Manag. Innov., 2013, 8(3):29-30

[12] Kim K Y. "Business Intelligence and Marketing Insights in an Era of Big Data. KSII Trans," Internet Inform. Syst., 2014, 8(2):567-582.

[13] Brandao A, Pereira E, Portela F, Santos M. "Realtime Business Intelligence platform to maternity care," Biomedical Engineering and Sciences, IEEE, 2014:79-384.

[14] Sun Y, Pei Z B, Wei W. "Design of Business Intelligence Front-end Tool Based on Mashup Framework," Comp. Mod., 2013, 1(9):219-225.

[15] Yang Z, Fong S, Fiaidhi J, Mohammed S. "RealTime Clinical Decision Support System with Data Stream Mining," J. Biomed. Biotech., 2012, 2012(5):580186.

[16] Ferretti V. "A Multicriteria Spatial Decision Support System Development for Siting a Landfill in the Province of Torino (Italy)," J. Multi-Crit. Decis. Anal., 2011, 18(5-6):231-252. 